\title{
Principales abréviations bibliographiques.
}

Akad. Vorles.

Amer. journ. scienc.

Annal. chem. et pharm.

Annal. chin. et phys.

Archiv u. Landesdurchforschung

v. Böhmen

Ber. d. D. Chem. Ges.

Bull. soc. chim. Paris

Bull. soc. minér. de Fr.

Centralb. f. Min.

Comp. rend. acad. scienc. Paris

Dentsch. Chem. Gesellschaft

Eng. et Min. Journal

Géol. Mag.

Journ. Amer. chim. soc.

Ber. d. Kgl. Bayer. Akad.

Minéral Mag.

Min. u. petr. Mitt.

N. Jahrb. f. Min.

Nova acta reg. soc. Upsala

Phil. magaz.

Phil. trans.

Proceed. Royal. soc.

Sitz. Ber. d. k. k. Akal.

Zeitsehr. d. B. g. g.

Zeitschr. d. D. geol. G.

Zeischr. f. snal. Chem.

Zeitschr. f. anorg. Chem.

Zeitschr. f. Baumaterialienkunde

Zeitschr. f. d. Ges. Naturwissenschaften

Zeitschr. f. Kryst.

Zeitschr. f. phys. Chem.

Wied. Annal.
$=$ Akademische Vorlesungen .

= American Journal of Science. New-Haven.

$=$ Annalen der Chemie und Pharmacie. Leipzig.

$=$ Annales de chimie et physique. Paris.

$=$ Arehiv und Landesdurchforschung von Böhmen.

$=$ Berichte der Deutschen Chemiker-Gesellschaft.

= Bulletin de la Société chimique de Paris.

= Bulletin de Société minéralogique de France. Paris.

= Centralblattfür Mineralogie,Geologie etc. Stuttgart.

= Comptes Rendus del'Académie des sciences. Paris.

$=$ Deutsehe Chemiker-Gesellschaft.

$=$ The Engineering and Mining Journal. New-York.

$=$ Geological Magazine. London.

= Journal of the american chemical Society. NewYork.

$=$ Berichte der Königlich Bayrischen Akademie.

= Mineralogical Magazine. London.

$=$ Mineralogische und petrographische Mitteilungen. Herausgegeben von G. Tschermak. Wien.

$\doteq$ Neues Jahrbuch für Mineralogie. Stuttgart.

= Nova acta regise societatis scientiarumUpsaliensis.

$=$ Philosophical Magazine.

$=$ Philosophical transactions of the Royal Society. London.

$=$ Proceedings of the Royal Society. London.

= Sitzungsberichte der k. k. Akademie der Wissenschaften zu Wien.

$=$ Zeitschrift der Bayrischen geolog. Gesellschaft.

$=$ Zeitschrift der Deutschen geologischen Gesellschaft. Berlin.

$=$ Zeitschrift für analytische Chemie, herausgegeben von Fresenius. Wiesbaden.

= Zeitschrift für anorganische Chemie. Hamburg.

= Zeitschrift für Baumaterialkunde. Berlin.

$=$ Zeitschrift für die gesamten Naturwissenschaften. Berlin.

$=$ Zeitschrift für Krystallographie und Mineralogie. Herausgegeben von P. Groth. Leipzig.

$=$ Zeitschrift für physikalische Chemie. Herausgegeben von W. Ostwald und J. H.van't Hoff.

$=$ Wiedemanns Annalen der Physik. 
